patient was a similar age to them and was local to the area. Students fed back in post-session evaluation that it had changed their perception of those with alcohol and drug problems.

Conclusion Some students did state a desire to still see patients for themselves; seeing patients personally will always remain important but we describe the use of a digistory as a powerful teaching tool to generate dialogue amongst learners, enhance knowledge exchange and address possibly misplaced attitudes to a vulnerable patient group. We recommend the use of digistories as a novel and effective teaching method to enable patients to tell very personal storeys whilst still protecting their anonymity.

Disclosure of Interest None Declared

\section{PTU-007 EVALUATING ENDOSCOPY TRAINERS; HOW RELIABLE ARE PEER EVALUATORS?}

doi:10.1136/gutjnl-2013-304907.100

1."L Macdougall, ' $\mathrm{S}$ Corbett, 'M Welfare, ${ }^{2} \mathrm{C}$ Wells, ${ }^{1} \mathrm{~J}$ R Barton. ${ }^{1}$ Northumbria Healthcare Trust, Newcastle-upon-tyne, ${ }^{2}$ North Tees Hospital, Stockton-upon-Tees, UK

Introduction The training of future endoscopists is important to ensure the ongoing provision of a safe endoscopy service within the UK; however endoscopy training is of a variable standard. Peer evaluation can be used to improve teaching but this does not currently routinely occur in local endoscopy units. We therefore wanted to assess the reliability of peer evaluations using an evaluation tool currently being developed to gain both trainee and peer evaluations.

Methods The DOTS tool has been developed using the list of attributes described by Wells ${ }^{1}$. In order to gain an assessment of reliability the tool was trialled on JAG approved Training the Trainer courses. Courses from November to March 2012 were contacted and asked to participate. Each course attendant was then sent an information letter and consent form. On day two of the course participants were asked to complete a copy of the DOTS for each of the training episodes they observed.

Data was analysed using SPSS 14; mean score and Cronbach alpha were calculated. Reliability was calculated using Generalisability theory; an initial analysis was performed using only trainers, peers and trainer: peer interaction as facets. A further analysis was then conducted including all possible sources of variance.

Results Eight of the ten courses contacted agreed to participate; all course participants consented to the study. 189 evaluations were collected; these were completed by 58 different peers; 45 trainers were evaluated receiving from one to ten evaluations each. Mean total evaluation score was 63.3 (out of 85); standard deviation 8.6. The tool showed a high level of internal consistency with a Cronbach alpha of 0.895 . In the initial analysis $44 \%$ of the variance of scores was explained by the difference in trainers' ability to teach, $35 \%$ due to peer variance and $21 \%$ by peer:trainer interaction. The G-coefficient for one rater was 0.44 and three raters were required for a G-coefficient of 0.7 . When the analysis was repeated the effect of course accounted for $20 \%$ of the variance in scores. Reliability was much lower with a $G$ coefficient of 0.28 for one rater.

Conclusion The DOTS tool showed a high level of internal consistency. On initial analysis only three peer reviewers were required to gain acceptable levels of reliability. However on reanalysis the effect of course was responsible for the $20 \%$ of the variance and if results were generalised across course then the tool showed poor reliability. The effect of course was unexpected and needs to be investigated further; the tool also needs to be trialled within local units.

\section{Disclosure of Interest None Declared}

\section{REFERENCE}

1. Wells, C., The characteristics of an excellent endoscopy trainer. Frontline Gastroenterology, 2010. 1: p. 13-18.

\section{PTU-008 SIMULATED ENDOSOPIC TRAINING: HOW JUNIOR DOCTORS UTILISE THIS RESOURCE}

doi:10.1136/gutjnl-2013-304907.101

1. ${ }^{*} \mathrm{M}$ G Keane, ${ }^{2} \mathrm{~L}$ Marelli, ${ }^{3} \mathrm{H}$ Potts, ${ }^{4} \mathrm{P}$ Berlingieri. ' Gastroenterology, Newham University Hospital; ${ }^{2}$ Gastroenterology, Homberton University Hospital NHS Foundation Trust; ${ }^{3} \mathrm{CHIME}$, UCL; ${ }^{4}$ Royal Free Hospital, London, UK

Introduction Simulation is increasingly being recognised as an attractive tool to support endoscopic training. Standard training has been associated with certain limitations; longer procedural times, cost, unpredictable pathology and occasionally patient dissatisfaction. Use of endoscopic simulators, has been suggested as alternative training method. Although advocated by national and international endoscopy societies (BSG, ASGE), when and how it should be incorporated into endoscopic training is still debated. A recent Cochrane review, suggested it was of most benefit to novice endoscopists.

An endoscopic simulation programme has been established at the Royal Free simulation centre since 2009. Completion of the course is not a formal speciality training programme requirement. Enrolled trainees progress through a staged curriculum with frequent assessment of their endoscopic competencies. How junior trainees use this resource was explored in this study.

Methods All trainees that have undertaken endoscopic training at the simulation centre between 2009 and 2012 were invited to complete an anonymous online questionnaire. Subsequently a targeted focus group was conducted; participants included trainees and simulation centre trainers.

Results 62 trainees were invited to complete the survey, with a response rate of $48 \%(30 / 62)$. The majority of trainees $(93 \% ; 28 / 30)$ completed the course during evenings or at weekends. $77 \%$ selffunded the course and just 7\% obtaining study leave. Trainees enrolled on the course for a median of 2 months. 52\% (15/29) were studying for a postgraduate exam while completing this course and 1 in 5 trainees commuted from outside the M25 to attend the course. A third of trainees were undertaking a rotation in Gastroenterology when they enrolled on the course and $82 \%(22 / 27)$ wanted to pursue a career in Gastroenterology. 68\% (17/25) reported they were actively applying for Gastroenterology or Surgical registrar training posts in the next 12 months. Frequently sited course outcomes by trainees included; greater familiarity with endoscopic equipment and technique, an opportunity to gain basic endoscopic skill training as a foundation doctor or $\mathrm{SHO}$, improved individual time management skills and was an opportunity for trainees to demonstrate a relevant example of commitment to speciality.

Conclusion Trainees completing this course sited a broad range of perceived learning outcomes. In addition to gaining endoscopic skill training, completing the course enabled trainees to develop their interpersonal skills and demonstrate commitment to speciality. This study supports junior doctors undertaking simulated endoscopic training.

Disclosure of Interest None Declared

\section{PTU-009 FROM ABSTRACT TO FULL PUBLICATION: A 15-YEAR REVIEW OF BRITISH SOCETY OF GASTROENTOROLOGY (BSG) CONFERENCE OUTCOMES}

doi:10.1136/gutjnl-2013-304907.102

."M Kurien, ' $A$ D Hopper, ${ }^{1} \mathrm{R}$ Sabbagh, ${ }^{1}$ A Beer, 'D S Sanders. 'Department of Gastroenterology, Royal Hallamshire Hospital, Sheffield, UK

Introduction Abstract presentations at scientific meetings allow rapid dissemination of novel research and enables peer review before submission for publication. Not all abstracts are subsequently published in peer reviewed journals. The likelihood of subsequent full publication of abstracts from other medical specialty meetings has 